\title{
II. Urkundliches zur Genealogie der Herzogin Judith von Bayern.
}

Von

Edmund Freiherru von Oefele, k. Reichsarchivrath.

Dass Herzog Arnulf von Bayern einen Sohn Namens Ludwig hatte, wird wohl Jedem klar, der eine langst bekannte Traditions. notiz des Klosters St. Emeram in Regensburg ${ }^{1}$ ) ohne Voreingenommenheit liest. Wenn nach derselben Judith, die Tochter Arnulfs and Wittwe Herzog Heinrichs I. von Bayern suna cum mana filii sui Heinricie ein Gut, welches sfrater eins Hluduvicase besass, an das Kloster gab mit dem Bedinge, dass ,ipsa domna et frater eius Hluduuicus e es lebenslănglich geniessen sollten, so ist eben Ludwig nicht Heinrichs II., sondern der Judith Bruder gewesen.

Um so mehr fällt es anf, dass Arnold, der in der ersten Hålfte des eilften Jahrbunderts tiber das Emeramskloster, welchem er angehörte, schrieb, der entgegengesetzten Meinung war ${ }^{2}$ ). Ihm vermuthlich folgend hat Aventin ${ }^{3}$ ) nicht unter Arnulfs, sondern unter Heinrichs I. Kindern einen Ludwig aufgefübrt, und in neuerer Zeit behauptete Janner 4 ), das ,frater eius c der Traditionsnotiz sei von einigen Genealogen falsch gedeutet worden; es könne damit nicht ein Bruder der Judith, sondern nur Heinrichs Bruder gemeint

1) Zuerst gedruckt bei Pez, Thesaur. anecdot. I c, 62 s., zuletzt bei Ried, Codex dipl. episcopat. Ratisbonens. I, 106; jetzt neu nach der Handscbrift als Beilage $I$.

7) Arnoldi liber $I 1$. de memoria beati Emmerammi et eius cultorum, cap. 57 (Mon. Germ. hist., Script. IV, 571): „Judita, Norici regni ducissa... pro se suisque filiis Hluduwico atque Heinrico."

7) Annales ducum Boiariae V, 1; Bayerische Chronik V, 5 (Sämmtliche Werke III, $16 ; V, 274)$.

4) Geschichte der Bisch fe von Regensburg I, 361, Anm. 1. 
sein, denu sonst hätte es nacb der Sprache jener Zeit nicht seiuse sondern *suus e geheissen.

Nun kommt freilich ein ungrammatischer Tausch zwischen pronomen demonstrativum, possessivum und reflexivum in Latein des Mittelalters haufig genug vor; aber er war keine strikte Regel, vorherrschend blieb der rechte Gebrauch. Wenn also. in jener Traditionsnotiz ssibi serviendor fehlerhaft stebt für ei serviendo und sadvocati suir fehlerhaft für advocati eius - denn Faramund ${ }^{1}$ ) ist offenbar der Hochstifts- und Klostervogt - so hindert das nicht, ein zweimal erscheinendes seius, in grammatisch richtigem Sinne aufzufassen.

Aber jeglichen $Z$ weifel schneidet ein anderes Schriftstück ans St. Emeram ab, das bisher noch nicht veröffentlicht wurde ). Es ist eine Carta traditionis, welche Judith dem Kloster ausstellt, als sie nach dem Hinscheiden Ludwigs die frühere Vergabung von ihrer beider Todes wegen in eine volle Schenkung nnter Lebenden verwandelt. Auch hier findet sich fehlerhaftes ssui * und ssibic, dagegen ist jede Verdunbelnng der Verwandtschaftsbeziehung ansgeschlossen durch die subjektive Fassung des Scbriftstückes. Wahrend die Notitia traditionis, objektiv gefasst, überall in der dritten Person spricht und so eine fulsche Beziehung des ersten eins begünstigen mag, wird in der Carta traditionis die Schenkerin redend eingeführt ${ }^{3}$ ), worauf sie in unmissverstăndlicher Weise Heinrichs II. als , filii mei c erwhnt und Ludwig als sfrater mense bezeichnet.

Dieser Ludwig war mittlerweile gestorben, die Worte der Carta ssicut in ultimis vitas suae temporibus possedite ergeben es zweifellos. Aber leider sind beide Schriftstücke ohne Datum, und nur annahernd lässt sich die Zeit der Akte bestimmen. Dass der zweite nicht um sehr viele Jabre spater fallt, darf man wohl ans der Identitat der Zeugen schliessen. Graf Sarhilo, der an ihrer Spitze steht, ist sonst im Jahre 973 beurkundet ${ }^{4}$ ). Ein fester

1) Vielleicht identisch mit dem Varamunt, der eine Tradition an St. Emerau zur Zeit Bischof Wolfgangs bezeugt, Quellen und Erörterungen zur Bayer. Gesch. I, 9.

3) S. Beilage II.

3) Die Namen der Zeugen sind auf Betrieb der Monche beigeftigt worden, desshalb tritt, wahrend Judith im Singular spricht, hier die plurale Redeweise (subscribere fecimus) ein.

) Mon. Germ. hist., Dipl. I, p. 586, Nr. 433; I, p. 50, Nr. 40. 
Punkt jedoch, der nach rückwärts die Gränze bildet, ist der Beginn der Waltung des Bischofes Wolfgang von Regensburg im Jahre 972. In des Bischofes Hand wird aber das Schenkungsobjekt aufgelassen, unter ihm also steht St. Emeram noch unmittelbar, es ist keine Rede von Ramwold, den er zuerst zum Propste, dann im Jahre 975 zum Abte des Klosters einsetzt. Vlelmehr sind Dompropst and Domdechant, die unter dem Bischofe das Kloster regierten ${ }^{1}$ ), bei der Besitzübertragung zugegen. Anderseits ist Heinrich II. noch Landesherzog, denn э cum manu Heinrici ducis × findet jedesmal die Auflassung statt. So fallen sicher die beiden Akte nicht nach 974, wo Heinrich sich empört und in die Verbaunung geschickt wird. Judiths Eintritt in das Kloster Niedermünster - sie beisst ja schon ssanctimonialis feminar - kann anch vor dieser Katastrophe geschehen sein. An das folgende Jahr, wo Heinrich, seiner Haft entronnen, karze Zeit wieder in Bayern weilt, Ramwold aber aus seinem Kloster flieht, ist nach der ganzen Lage der Dinge, besonders dem Verfahren des Regensburger Klerus gegen Heiurïch, nicht za denken.

Dass Arnold vou St. Emeram, obwohl er anch die Carta traditionis kannte, denn er spricht von ihrer Rückfallsklausel, doch die Verwandtschaft zwischen Judith und Ludwig unrichtig angibt, ist eben einer von jenen Flüchtigkeitsfehlern, wie sie das Mittelalter in genealogischen Dingen, sobald ein paar Generationen in Mitte lagen, so leicht beging. War doch auch Kaiser Heinrich II. im Jahre 1021 der irrigen Meinung, sein Grossvater sei es gewesen, der Aiterhofen dem Kloster verliehen habe \%.

Herzogin Judith bat aber dennoch einen zweiten Sohn gehabt, nur hiess derselbe uicht Ludwig, sondern Brun. Das erfahren wir ebenfalls durcb eine Traditionsnotiz, diessmal aus Niedermünster, wo sie in ein Salbuch ${ }^{3}$ ) abgeschrieben wurde und so erhalten blieb. Nach derselben verlieh ein Herzog Heinrich scum mana matris sue Juditec dem Kloster Niedermünster den Niessbrauch eines Gutes, welches »frater eius nomine Brunc in Beutelhausen besessen

1) Einen andern Propst und Dechant kann Arnold (M. G. SS. IV, 559) mit den Worlen "Prepositus et decanus monasterium regebant post episcopum" nicht wobl meinen. Auch Andreas Mayer fuhrt im Thessur. nov. iur. ecclesiast. II, 81, 91 Rihber nnd Walther als Dompropst und Domdechant auf. ๆ) Mon. Boic. XXVIIl a, 491.

3) Herausgegeben von Studienrektor Hoger im XXIII. Bande der Ver- 
hatte. Unter seiusc ist ohne $Z$ weifel Heinrich zu verstehen; die Mitwirkung der Matter dürfte anf Vormundschuftsführung denten; Brun scheint todt gewesen zu sein. Der erste Heransgeber bat nun gemeint, smatris" sei verschrieben, es müsse saviae s heissen, Heinrich sei der spätere Kaiser, Brun dessen Bruder, der Augsburger Bischof. Eine durch Nichts begrïndete Konjektur ')! Solches Verschreiben eines Zeitgenossen, so zu sagen in einem über die Handlung aufgenounmenen Protokolle, ist höchst unwahrscheinlich. Einem Kopisten aber fiel es schwerlich ein, zweimal , matris zu schreiben, wenn seine Vorlage saviae, hatte. Dann ergaben sich anch chronologische Schwierigkeiten. Von Judith hören wir zuletzt im Jaure $980^{\text {g)}}$; Heinrich IV. wird Mitregent seines Vaters kaum vor Beginn der neunziger Jahre, alleiniger Herzog im Jahre 995. Oder wie liesse es sich erklären, dass Brun nicht mebr im Besitze des Gutes ist? Und dieses selbst, was ist es Anderes als das Krongut Butileshusa, das die Kaiser Otto I. und Otto II. im Jahre 973 auf Bitten der Judith dem Kloster Niedermünster schenkteu ? ${ }^{9}$ Hat also ein Herzog von Bayern, was er an dem Gute besass and vergeben konnte, dem Kloster übertragen, so muss Dieses früher geschehen sein. Durch die volle kaiserliche Schenkung wurde die blosse Niessbrauchverleihung des Herzogs bestätigt.

Ob Brun der Herzogin Judith Sohn auch jener Bruno gewesen, der im Jahre 976 von Kaiser Otto II. snepos nosters genannt wird, aber, wie es scheint, nicht mehr am Leben war ${ }^{4}$ ), bleibe dahingestellt.

\section{Beil agen.}

I.

Traditio venerande ac sanctae monialis femine Iuditae.

Agnoscat igitur ounnium fidelium industria, qualiter venerabilis patrona ac sanctimonialis fenina Iudita nuncupata, memor inmarcescibilis remune-

handlungen des historischen Vereins für Niederbayern 1885; die Traditionsnotiz auf Seite 275-276. S. jetzt unten Beilage III.

1) Vielleicht ist es schon ein alterer Irrthum, denn jene Verse auf die Fundatrices von Niederminnster, welche vorne in dem Salbuche stehen, erwhbnen 2weier junger "nepotes" (Enkel?) der Judith.

2) M. G. DD. II, p. 258, Nr. 230.

9) Ibid. I, p. 585, Nr. 432; II, p. 51, Nr. 41.

4) Ibid. II, p. 155, Nir. 138. 
rationis et eternae beatitudinis, tradidit ana cum manu filii sui Heinrici ducis ad sanctum Emmerammum et ad servitium monachorum talem proprietatem, qualem frater eius Hluduricus in loco Fitarahoue sibi serviendo in potestate habuit, cum omnibus rebus ad eundem locum iuste et legitime pertinentibus; hoc est curtem cum edificiis et reliquis curtilibus, villis et casis, vineis ${ }^{1}$ ) ac vinitoribus et aurariis ${ }^{2}$ ). agris, pascuis, silvis, aquis, piscationibus, molendinis, mobilibus et inmobilibus, exitibus et reditibus, quesitis et inquirendis omnique legitima cautione, mancipiis utriusque sexus et reliqua. Omnia \%, nt supra dictuu est, tradidit memorata domna ad altare sancti Emmerammi et ad commune servitium monachorum in manus Wolfgangi ${ }^{4}$ ) episcopi et adrocati sui Faramundi; $e^{3}$ ) quoque tenore, ut ipsa domna et frater eius Hludnuicus in servitium habuerint et utantur usque ad finem vitae suse, post ambornn vero obitum provenerit et redierit ipse locus in ius et servitium aecclesiae et fratrum cum omni integritate sine omnium contradictione. Huius traditionis testes sunt per aures tracti: Sarhilo comes, Theomar, Tagini, Anno, Unaltheri,.,) Timo, Papo, Ródperht, Engilmar, Egilolf, Tuoto, Orendil, Ysanrih, Uuirunt, Leoparto, Uuichram, Ógo, Ódalrih, Heinpreht, Gotedieo, Reginpreht, Purchart. Vestituram huins rei Timo presentaverat ad aram sancti Emmerammi et fratribus. Presentes quoqne tunc aderant: Rihherus prepositus, Unaltherus decanus, Iob, Amanoldus, Adalpertus et ceteri omnes.

Abschrift des zwolften Jahrbunderte in einem 'L'ı aditionskodex des Klusters St. Fmersm in Regensburg, Nr. 51/s der Literslien desselben im k. allgemeinen Reichsarchive (alte Bezeichnung: Z. 31), Blatt $55^{\prime}-56$.

\section{II.}

Traditio Heinrici ducis et Indithae matris eius.

Noverit omoium fidelinm, praesentium videlicet et futarorum, industria, qualiter ego Iudita pro remedio animae meae et parentum meorum cogitans tradidi ad dei servitium et sancti Emmerammi martyris tale praedium, quale visa sum habere in loco, qui dicitur Eitarhona, cum mann filii mei Heinrici ducis Bauuariorum cum omnibus ad eandem curtem inre et legitime pertinentibus, agris, pascuis, villis et casis, vineis ac vioitoribus et aurariis, silvis, aquis, piscationibus, molendinis, mobilibus et inmobilibus, quesitis et inquirendis, exitibus et reditibus, mancipiis quoque utriusque sexus et omnino onnibus, ut dixi, ad eundem locum inste pertinefntibus, et sicut frater mens Hludouuious in altimis vitae suae temporibus ad usus proprios possedit, in

1) veneis $\mathrm{Hs}$.

9) aureariis $\mathrm{HB}$.

8) In der Hв. steht zwischen reliqua und omnia keine Interpunktion.

4) Alsbald durch Ueberschreibung des f korrigirt aus Wolgangi.

5) Ea Hs.

9) Alsbald durch Ueberschreibuny des $h$ sorrigirt aus Uualteri. 
manum venerabilis viri Unolfgengi ${ }^{1}$ ) episcopi et Faramundi adrocati sui; ea scilicet ratione, ut monachi ibidem deo et sancto martyri eius Emmerammo servientes ad communem utilitatem perpetualiter eandem curtem possideant. Si vero aliquis episcoporum per futura tempora succedentium ad suum, quod fieri non credo, privatum servitium redigere vel vassallis suis ad beneficium dare temptaverit, heres meus sicut reliqua sibi derelicta haereditario iure et hanc curtem possideat. Fit ut haec traditio in perpetunm semper firma permaneat, hos testes, qui ea viderunt fieri et audierunt, subscribere fecimus, more legali per aures attractos: ${ }^{2}$ ) Sarhilo comes, Teomar, Tagani, Anno, Vualtheri, Timo, Papo, Rodpreht, Engilmar, Egilolf, Tuoto, Orendil, Ysanrih, Vuiront, Lieoparto, Ógo, Ȯalalrich, Heimpreht, Gotidieo, Reginpreht, Puracbart, Vuiram.

A bschrift des eilften Jahriunderts in einem Traditionskodex des Klogters St. Emeram in Regensburg, Nr. $5^{1 / 2}$ der Literalien desselben im $\mathbf{k}$. allgemeinen Reichaarchive (alte Bezeichnung: Z. 32), Blatt 35'-36'.

\section{III.}

Traditio Heinrici ducis et matris eins Ivdite.

Monet divina pietas omnes scire volentes, presentes videlicet et futuros, qualiter nobilissimus dux nomine Henricvs cum manu matris sue domne Ivdite tradidit ad aram sancte Marie tale predium, quale frater eius nomine Brpn habere visus est in loco, qui dicitur Pewtelshausen ${ }^{3}$ ), pro remedio anime sue et omnium parentum suorum; ea videlicet racione, at ad usum et stipendium sanctimonialium et clericorum ibidem servientium perpetuo consistat, ita ut nemo potestatem habeat, sine consensu sanctimonialiom iam dictum locum in beneficium dare aut commutare, sed cum omnibus ad iam scriptum predium pertinentibus, edificiis, agris, vineis, quesitis et inquirendis cunctisque ibidem iuste pertinentibus perenniter consistendum et sanctis monialibus perfruendom. Hanc tradicionem suscepit advocatus Piligrim. Et isti sunt testes: Poppo comes, frater eius Aribo, Chaldalhoch "), Arnol "), Werinheri, Waltchuon, Chadalhoch; Arnolt, Helmpret, Reginpret, Pabo, Meginhart, Pero, Ruodprecht, Ógo, Egilof, Adalo.

Abschrift des fünfzehnten Jahrhunderts, wobei zum Theile eine altere Scbrift nachgeahmt ist, im „Saal- und Fundations-Buch" des Reichsotiftes Niedermunster zu Regensburg vom Jahre 1444 (jetzt im k. Kreisarchive Landshut), Blatt 38' vor dem auf Beutelচausen bezúglichen urbarialen Eintrage.

1) So die Hs.

) attracti $\mathrm{Hs}$.

3) Diese Namensform des fünfzehnten Jahrhunderts gebraucht der $\mathbf{A b}$ schreiber wohl aus dem praktischen Grunde der Verstăndlichkeit.

4) So die Hs. 